\title{
Qubit thermodynamics far from equilibrium: two perspectives about the nature of heat and work in the quantum regime
}

\author{
Andrés Vallejo, Alejandro Romanelli, and Raúl Donangelo \\ Facultad de Ingeniería, Universidad de la República, Montevideo, Uruguay
}

(Dated: March 17, 2021)

\begin{abstract}
Considering an entropy-based division of energy transferred into heat and work, we develop an alternative theoretical framework for the thermodynamic analysis of two-level systems. When comparing these results with those obtained under the standard definitions of these quantities, we observe the appearance of a new term of work, which represents the energy cost of rotating the Bloch vector in presence of the external field that defines the local Hamiltonian. Additionally, we obtain explicit expressions for the temperature, the heat capacity and the internal entropy production of the system in both paradigms. In order to illustrate our findings we study, from both perspectives, matter-radiation interaction processes for two different systems.
\end{abstract}

\section{INTRODUCTION}

Quantum physics is an intrinsically dynamic theory, and therefore time-dependence is essential in its description. Classical thermodynamics, on the other hand, mostly considers closed systems that evolve quasiadiabatically [1]. For this reason, when we insert quantum dynamics into thermodynamics we obtain a quantum version of finite-time thermodynamics, which is intimately related with the theory of open systems 2].

Heat and work are the basic mechanisms of energy exchange between thermodynamic systems. From the classical point of view, heat is usually defined as the energy flow which occurs exclusively due to the temperature difference between the systems. Work, on the other hand, is the energy exchange which can be measured through the variation of a macroscopic parameter, such as the volume of the system $[1,3-6]$.

Although for classical thermodynamic systems the classification of the energy transfers as heat and work is not free from controversy, the situation is even more complex when quantum systems are considered. Many nonequivalent definitions of these quantities can be found in the literature $7-13$, so the correct identification of heat and work in that regime can be considered an open problem.

One of the most extensively considered paradigms regarding these quantities was proposed by Alicki several decades ago [13]. Defining the internal energy of the system as the expected value of the local Hamiltonian $H$ in the actual reduced state $\rho$,

$$
E=\langle H\rangle=\operatorname{tr}[H \rho],
$$

thus, an infinitesimal energy change takes the form

$$
d E=\operatorname{tr}[d H \rho]+\operatorname{tr}[H d \rho] .
$$

The first term on the right-hand side of Eq. (2) is the energy change due to changes in the Hamiltonian of the system, associated to some control parameter which can be modified by the experimenter. Considering the previous discussion about the classical work made on the system, it is reasonable to define the infinitesimal of work as

$$
\delta \mathcal{W}=\operatorname{tr}[d H \rho] .
$$

Thus, in order to assure the validity of the first law, the infinitesimal of heat is defined as

$$
\delta \mathcal{Q}=\operatorname{tr}[H d \rho] .
$$

Therefore, from this point of view, heat is related to changes in the density matrix describing the quantum state.

Despite its wide application in several contexts 14, 15], this approach has some weak points. For example, if we consider two interacting systems with constant local Hamiltonians, the above equations implies that, even if work is done on the global system through a timedependent Hamiltonian, no work is done on the individual systems, which is counterintuitive.

In this paper we will explore some consequences of a recent proposal that claims to resolve these issues [16, 17]. It is based on the hypothesis that the von Neumann entropy is a valid extension of the thermodynamic entropy in the quantum regime, and on the fact that it depends only on the eigenvalues $\lambda_{j}$ of the density matrix $\rho_{S},[18]$ :

$$
S_{v N}=-\sum_{j=1}^{N} \lambda_{j} \ln \lambda_{j} .
$$

This implies that the changes in $S_{v N}$ are always accompanied by changes in the eigenvalues of $\rho_{S}$. But classically, the entropy change is proportional to the reversible heat transfer. These observations suggest that heat could not be related with changes in the whole density matrix (see Eq. (4)), but only on its eigenvalues.

The remainder of this paper is organized as follows. In Section II we introduce the new notions of work and heat for a generic finite-dimensional quantum system. In Section III, using the above ideas, we develop the complete thermodynamics for a two-level system and we compare the results with those which arise from assuming Alicki's theoretical framework [19]. As we shall see, the simplicity of the two-level system allows a very simple geometric 
interpretation of the thermodynamic quantities. In Section IV, we perform both thermodynamic analyses for two specific matter-radiation interaction processes. Some conclusions are presented in Section V.

\section{THE NEW PARADIGM}

In this section we briefly explain the ideas behind the new proposal. First, we note that, in the standard paradigm, Eqs. (3) and (4) are equivalent to,

$$
\delta \mathcal{W}=\sum_{j} \rho_{j j} d E_{j}
$$

and

$$
\delta \mathcal{Q}=\sum_{j} d \rho_{j j} E_{j}
$$

where $\left\{E_{j}\right\}$ are the eigenenergies of the system, and $\left\{\rho_{j j}\right\}$ their corresponding probabilities. These expressions are equivalent to those corresponding to the classical notions of work and heat for systems with a discrete spectrum. But it would be reasonable that quantum features, such as the existence of coherence between the different eigenstates, play an important role, which is not covered by the previous description [20, 21].

To analyze the problem from a different perspective, we start by writing the instantaneous spectral decomposition of the density matrix,

$$
\rho=\sum_{j} \lambda_{j}\left|\psi_{j}\right\rangle\left\langle\psi_{j}\right|
$$

where $\left\{\left|\psi_{j}\right\rangle\right\}$ are the eigenfunctions and $\left\{\lambda_{j}\right\}$ the set of corresponding eigenvalues. The above equation, together with Eq. (1), allows to express the internal energy as,

$$
E=\sum_{j} \lambda_{j}\left\langle\psi_{j}|H| \psi_{j}\right\rangle
$$

so the infinitesimal energy change is given by

$$
d E=\sum_{j} d \lambda_{j}\left\langle\psi_{j}|H| \psi_{j}\right\rangle+\sum_{j} \lambda_{j} d\left\langle\psi_{j}|H| \psi_{j}\right\rangle .
$$

Recalling Eq. (5), it is clear that only the first term on the right-hand side of the above equation is linked to the entropy change. Thus, it is the only term which should be considered as heat, so we define,

$$
\delta Q=\sum_{j} d \lambda_{j}\left\langle\psi_{j}|H| \psi_{j}\right\rangle
$$

and, as a consequence,

$$
\delta W=\sum_{j} \lambda_{j} d\left\langle\psi_{j}|H| \psi_{j}\right\rangle
$$

Note that in this new paradigm, work is related not only to the possibility of driving the Hamiltonian, but also with the change in the eigenvectors of the density matrix. Of course, for thermal equilibrium states, and, more in general, for any incoherent state in the energy basis, the Hamiltonian and the density matrix commute. Thus, $\lambda_{j}=\rho_{j j},\left\langle\psi_{j}|H| \psi_{j}\right\rangle=E_{j}$, and, as a consequence, both paradigms are equivalent in that limit.

In the next section we focus on the study of two-level systems, a case which is interesting in its own right due to its technological applications, and which, due to its simplicity, allows to obtain a clear geometrical interpretation of the thermodynamic quantities.

\section{THERMODYNAMIC QUANTITIES FOR TWO-LEVEL SYSTEMS IN THE BLOCH VECTOR REPRESENTATION}

\section{A. Internal energy, heat and work}

A convenient way to visualize the state of a two-level system is through its Bloch vector,

$$
\vec{B}=\left(B_{x}, B_{y}, B_{z}\right)
$$

whose components are, aside from a factor $\hbar / 2$, the expected values of the spin operators $S_{x}, S_{y}$ and $S_{z}$,

$$
\begin{aligned}
& B_{x}=\left\langle S_{x}\right\rangle=\operatorname{tr}\left(\rho_{S} \sigma_{x}\right), \\
& B_{y}=\left\langle S_{y}\right\rangle=\operatorname{tr}\left(\rho_{S} \sigma_{y}\right), \\
& B_{z}=\left\langle S_{z}\right\rangle=\operatorname{tr}\left(\rho_{S} \sigma_{z}\right),
\end{aligned}
$$

where $\sigma_{x}, \sigma_{y}$ and $\sigma_{z}$ are the Pauli matrices. On the other hand, aside from an irrelevant scalar multiple of the identity, a generic Hamiltonian in two dimensions adopts the form,

$$
H=-\vec{v} \cdot \vec{\sigma}
$$

where $\vec{v}$ can be associated to an effective magnetic field, and $\vec{\sigma}$ is a formal vector whose components are the Pauli matrices. In terms of the Bloch vector, the density matrix of a two-level system can be written as,

$$
\rho=\frac{1}{2}[1+\vec{B} \cdot \vec{\sigma}]
$$

Using Eqs. (1), (15), (16) and the identity

$$
(\vec{a} \cdot \vec{\sigma})(\vec{b} \cdot \vec{\sigma})=(\vec{a} \cdot \vec{b}) I+i \vec{\sigma} \cdot(\vec{a} \times \vec{b})
$$

we obtain the internal energy

$$
E=-\vec{B} \cdot \vec{v}
$$

From the above equation we can write an infinitesimal energy change as:

$$
d E=-d \vec{B} \cdot \vec{v}-\vec{B} \cdot d \vec{v} .
$$




\section{Standard framework: Paradigm 1}

In the standard framework, work is performed on the system only if the Hamiltonian is time-dependent. Heat, on the other hand, is related to changes in the quantum state. This point of view leads to the following natural definitions of infinitesimal work and heat for a two-level system,

$$
\begin{aligned}
& \delta \mathcal{W}=-\vec{B} \cdot d \vec{v}, \\
& \delta \mathcal{Q}=-d \vec{B} \cdot \vec{v},
\end{aligned}
$$

in such a way that the first law has the same structure than in the classical case: $d E=\delta \mathcal{Q}+\delta \mathcal{W}$.

From this point of view, the work is zero when the effective magnetic field is constant in time, or when its change is orthogonal to the Bloch vector, i.e, orthogonal to the instantaneous magnetization. Conversely, heat is zero when the Bloch vector is constant in time, i.e. the system is in equilibrium, and also when its change is orthogonal to the effective magnetic field. This situation includes the special case in which the qubit evolves unitarily.

\section{Alternative treatment: Paradigm 2}

To analyze the problem from this new perspective, we first note that the eigenvalues of $\rho_{S}$ can be written as

$$
\lambda_{+/-}=1 / 2 \pm B / 2,
$$

where $B$ is the modulus of the Bloch vector. Therefore, considering Eq. (5), we can write the von Neumann entropy of the qubit as,

$$
\frac{S_{v N}}{k_{B}}=-\left(\frac{1+B}{2}\right) \ln \left(\frac{1+B}{2}\right)-\left(\frac{1-B}{2}\right) \ln \left(\frac{1-B}{2}\right) .
$$

We note that in the case of two-level systems, the entropy depends only on $B$, so its changes are linked solely to changes in $B$. Thus, from the point of view according to which heat is associated to the energy exchange responsible for entropy change, we conclude that $\delta Q$ is different from zero if and only if $B$ changes.

If we write the energy of the system, Eq. (18), as

$$
E=-B \hat{B} \cdot \vec{v}
$$

where $\hat{B}$ is a unit vector in the direction of $\vec{B}$, the energy change can be partitioned as

$$
d E=-d B(\hat{B} \cdot \vec{v})-B d(\hat{B} \cdot \vec{v}) .
$$

As a result of the previous considerations, and since only the first term contributes to the entropy change, we define the heat and work exchanged in this second approach as

$$
\delta Q \equiv-d B(\hat{B} \cdot \vec{v})
$$

and

$$
\delta W \equiv-B d(\hat{B} \cdot \vec{v}) .
$$

It is interesting to consider in detail these expressions. From Eq. (27), we notice that the work done on the system is the product of the modulus of the magnetization $B$ and the infinitesimal change in the projection of the effective magnetic field on the direction of the magnetization,

$$
\delta W=-B d(|\vec{v}| \cos \theta),
$$

where $\theta$ is the angle between $\hat{v}$ and $\hat{B}$. Thus, in this framework, work can be performed on the system even if the Hamiltonian, i.e., $\vec{v}$, is fixed, provided that $\hat{B}$ changes in such a way that the angle between both vectors is not constant in time. In particular, work is extracted from the system, $\delta W \leq 0$, when the Bloch vector changes in such a way that $\hat{B} \cdot \vec{v}$ increases in time, i.e. when $\vec{B}$ tends to align itself with the effective field $\vec{v}$.

On the other hand, there are two kind of processes for which no heat is exchanged: 1 ) isoentropic processes, for which $B$ is constant, and 2) processes for which $\hat{B} \perp \vec{v}$ along the process. This situation includes states that encompass all possible entropy values, as long as the point representing the reduced state in the Bloch sphere moves on a plane orthogonal to the effective magnetic field. The states located on this plane are the statistical mixtures of the cat-states (SMCS) of the instantaneous Hamiltonian, and all of them possess zero energy, so if the system transits among these states the work exchanged is also zero.

Regarding the sign of the heat, it is clear that if the angle $\theta$ between $\hat{B}$ and $\vec{v}$ satisfies $\theta<\pi / 2$, the injection of heat into the system, $\delta Q>0$, implies an increase in entropy. Conversely, if $\theta>\pi / 2$, injection of heat leads to an entropy decrease. This suggests that temperatures associated to states in the upper hemisphere of the Bloch sphere take positive values, while those on the other hemisphere have opposite sign. This point which will be studied in detail in the next subsection.

\section{Discussion}

The relation between heat and work in the first paradigm, $\mathcal{Q}$ and $\mathcal{W}$, and in the second, $Q$ and $W$, can be obtained as follows. From Eq. (27),

$$
\delta W=-B d(\hat{B} \cdot \vec{v})=-B d \hat{B} \cdot \vec{v}-B \hat{B} \cdot d \vec{v},
$$

and since $-B \hat{B} \cdot d \vec{v}=-\vec{B} \cdot d \vec{v}=\delta \mathcal{W}$, we obtain:

$$
\delta W=\delta \mathcal{W}-B d \hat{B} \cdot \vec{v} .
$$

Similarly, the relation between the heats exchanged in both paradigms is:

$$
\delta Q=\delta \mathcal{Q}+B d \hat{B} \cdot \vec{v}
$$


Eq. (30) can be written as

$$
\delta W=\delta \mathcal{W}+\delta W^{\prime},
$$

where

$$
\delta W^{\prime}=-B d \hat{B} \cdot \vec{v} .
$$

We notice that the work associated to an infinitesimal process in paradigm 2 adds, to the standard contribution $\delta \mathcal{W}$ due to Hamiltonian driving, the additional term $\delta W^{\prime}$, which is related to the time variation of the density matrix eigenvectors in Eq. (12). Choosing the $z$ axis in the direction of $\vec{v}$, and expressing $d \hat{B}$ in spherical coordinates,

$$
d \hat{B}=d \theta \hat{e}_{\theta}+\sin \theta d \varphi \hat{e}_{\varphi}
$$

we find that

$$
\delta W^{\prime}=B \sin \theta \varepsilon d \theta,
$$

where $\varepsilon=|\vec{v}|$ is the positive energy eigenvalue.

Since the components of the Bloch vector are proportional to the expected values of the spin operators, $\vec{B}$ can be interpreted as the average magnetic dipole moment of the system, which is embedded in an external magnetic field $\vec{v}$. For a classical dipole, the potential energy is given by $U=-\vec{B} \cdot \vec{v}$, so it coincides with our expression for the internal energy, Eq. (18). Therefore, the work that must be performed against the magnetic field in order to rotate the dipole from the initial to the final configuration equals the potential energy difference between those two configurations.

In our case we notice that, unlike what occurs in unitary evolutions, the interaction with the environment drives the system along trajectories such that the polar angle $\theta$ may vary in time, which implies that rotational work must be performed against the magnetic field, in an amount:

$$
\delta W_{\text {rot }}=-\vec{M} \cdot \overrightarrow{d \theta}
$$

where $\vec{M}=\vec{B} \wedge \vec{v}$ is the torque exerted by the magnetic force, and $\overrightarrow{d \theta}=d \theta \hat{e_{\varphi}}$. It is now straightforward to show that $\delta W^{\prime}=\delta W_{\text {rot }}$. Thus, $\delta W^{\prime}$ is the energetic cost of rotating the dipole in the presence of the external field.

We also notice that $B \sin \theta$ is the coherence of the state measured using the $l_{1}$ norm, $C_{l_{1}}$ [22],

$$
C_{l_{1}} \equiv \sum_{i \neq j}\left|\rho_{i j}\right|=B \sin \theta
$$

From the above equations, we obtain

$$
\delta W^{\prime}=C_{l_{1}} \varepsilon d \theta
$$

so we conclude that for a fixed local Hamiltonian, work can be performed on the system only if coherence in the energy eigenbasis is present. In fact, from Eq. (38) we see that $C_{l_{1}}$ can be interpreted as the lever arm of the torque, revealing, in the context of the present work, the role of quantum coherence as a resource for thermodynamic tasks [23, 24]. Reciprocally, in Ref. [25] it is shown, employing a differential geometry approach, that the creation of coherence is detrimental to efficiency in finite-time thermodynamic processes.

As a simple example let us consider a qubit undergoing a pure dephasing process. Since the Hamiltonian is fixed, from the point of view of paradigm 1 no work is performed. Since the non-diagonal terms tend to zero while the populations remain constant, the Bloch vector evolves in such a way that the energy of the system does not change. This implies that no heat is exchanged either, so from the point of view of paradigm 1, pure dephasing is a non-dissipative process in which the information contained in the coherence is transferred from the system to the environment.

However, information possesses an energy value 26 28], so it should be expected that, despite maintaining its energy constant, the potential of the qubit to do work would decrease. This fact can be explained in a natural way analyzing the problem from the point of view of paradigm 2. Since during pure dephasing the entropy of the qubit increases, for positive temperature states, heat is transferred to the system. But since the energy of the system is constant, an equal but opposite amount of work is performed on the environment. This decrease in the ability to perform work can then be interpreted, as the result of giving some high quality energy (work), receiving, in exchange, the same amount of low quality energy (heat).

\section{B. Temperature}

Our main objective so far has been to compare the notions of heat and work within each of the two paradigms considered, However, the adoption of either one allows us to extend, in the case of two-level systems, other thermodynamic quantities to the quantum regime, considering their corresponding classical analogous concepts.

Temperature is clearly defined only for systems in thermodynamic equilibrium. Nevertheless, many definitions of temperature have been shown to be useful in nonequilibrium situations [29 37].

The temperatures that we define below should be interpreted as a measure of the entropy changes produced by the heat exchanged when the system finds itself in a particular state. They are not necessarily linked to the direction of the heat flow when thermodynamic systems are put in thermal contact. In fact, it has been theoretically predicted and experimentally shown that the direction of the heat flow between quantum systems in local thermal states can be reversed if quantum correlations are present in the initial state [38, 39]. 


\section{Paradigm 1}

Consistently with the classical case, we define the temperature of a two-level system as the derivative of the von Neumann entropy with respect to energy in a zero work process, a condition which is satisfied in the standard framework if the Hamiltonian is time-independent. Since the Hamiltonian is determined by the effective magnetic field $\vec{v}=\varepsilon \hat{v}$, fixing the direction $\hat{v}$ we define

$$
\frac{1}{\mathcal{T}}=\left.\frac{\partial S_{v N}}{\partial E}\right|_{\varepsilon}
$$

We observe that $S_{v N}$ depends only on $B$, which, in turn, depends on three arbitrary orthogonal components of the Bloch vector. Due to Eq. (1), the energy depends only on the component parallel to $\hat{v}$,

$$
E=-\varepsilon \vec{B} \cdot \hat{v}
$$

so we have:

$$
\frac{1}{\mathcal{T}}=\left.\frac{d S_{v N}}{d B} \frac{\partial B}{\partial(\vec{B} \cdot \hat{v})} \frac{\partial(\vec{B} \cdot \hat{v})}{\partial E}\right|_{\varepsilon} .
$$

From Eq. (23),

$$
\frac{d S_{v N}}{d B}=-k_{B} \tanh ^{-1}(B)
$$

and the other factors are

$$
\frac{\partial B}{\partial(\vec{B} \cdot \hat{v})}=\hat{B} \cdot \hat{v} ;\left.\quad \frac{\partial(\vec{B} \cdot \hat{v})}{\partial E}\right|_{\varepsilon}=-\frac{1}{\varepsilon} .
$$

Thus,

$$
\mathcal{T}=\frac{\varepsilon}{k_{B}(\hat{B} . \hat{v}) \tanh ^{-1}(B)} .
$$

We notice that pure states have zero temperature, except for those such that the Bloch vector is orthogonal to the effective magnetic field, for which the temperature is not defined. In the case of mixed states, the temperature diverges as the magnetization in the direction of $\hat{v}$ goes to zero. This behavior is similar to that corresponding to classical spin systems [40].

\section{Paradigm 2}

From this new perspective, a zero-work process is implemented keeping constant the product $\hat{B} \cdot \vec{v}$, so we define

$$
\frac{1}{T}=\left.\frac{\partial S_{v N}}{\partial E}\right|_{\hat{B} \cdot \vec{v}}
$$

or, equivalently,

$$
\frac{1}{T}=\left.\frac{d S_{v N}}{d B} \frac{\partial B}{\partial E}\right|_{\hat{B} . \vec{v}}
$$

In this case it is convenient to write Eq. (10) in the form

$$
B=-\frac{E}{\hat{B} \cdot \vec{v}}
$$

so that the second factor in Eq. (46) is written

$$
\left.\frac{\partial B}{\partial E}\right|_{\hat{B} \cdot \vec{v}}=-\frac{1}{\hat{B} \cdot \vec{v}}
$$

and, therefore,

$$
T=\frac{\varepsilon \hat{B} \cdot \hat{v}}{k_{B} \tanh ^{-1}(B)} .
$$

Two families of zero-temperature states appear in paradigm 2: those with $B=1$ (pure states), and $\hat{B} \cdot \vec{v}=0$ (SMCS states). On the other hand, the only infinitetemperature state is the maximally mixed state.

\section{Discussion}

First, we note the different position of the factor $\hat{B} . \hat{v}$ in Eqs. (44) (first paradigm) and in Eq. (49) (second paradigm). This implies that the relation between both temperatures is

$$
T=\mathcal{T}(\hat{B} . \hat{v})^{2}=\mathcal{T} \cos ^{2} \theta
$$

from which we deduce that they have always the same sign and that, for all possible states, $T \leq \mathcal{T}$. In particular, for incoherent states in the energy eigenbasis $(\theta=0)$ both temperatures coincide:

$$
T=\mathcal{T}=\frac{\varepsilon}{k_{B} \tanh ^{-1}(B)}
$$

If additionally the system reaches thermal equilibrium with an environment at temperature $T_{E}$, the reduced state is described by the Gibbs state in which the populations of the ground and the excited levels $P_{g}^{e q}$ and $P_{e}^{e q}$ are fixed by the environment temperature [41],

$$
T_{E}=\frac{2 \varepsilon}{k_{B} \ln \left(\frac{P_{g}^{e q}}{P_{e}^{e q}}\right)}
$$

Since in this case the Hamiltonian and the density matrix commute, the populations and the eigenvalues of the density matrix coincide. Therefore, from Eq. (22), we obtain,

$$
\ln \left(\frac{P_{g}^{e q}}{P_{e}^{e q}}\right)=\ln \left(\frac{1+B^{e q}}{1-B^{e q}}\right)=2 \tanh ^{-1}\left(B^{e q}\right) .
$$

Finally, from Eqs. (51), (52) and (53), we conclude that, in thermal equilibrium,

$$
T=\mathcal{T}=T_{E} .
$$





FIG. 1: Isothermal surfaces in the Bloch sphere, corresponding to the temperature values $k_{B} T_{1}=\varepsilon$ (red, upper region) and $k_{B} T_{2}=-2 \varepsilon$ (blue, lower region) in paradigm 1 (left panel) and paradigm 2 (right panel).

Therefore, at least in principle, both expressions (44) and (49) extend naturally the concept of temperature to the non-equilibrium situation.

We have already noted that in the context of paradigm 2 , the zero-energy plane divides the Bloch sphere in two hemispheres with opposite values of temperature. This is also true in paradigm 1 , since both temperatures have the same sign. This can be seen explicitly finding the energy-temperature relation from Eqs. (40) and (49),

$$
T=-\frac{E}{k_{B} B \tanh ^{-1}(B)}
$$

Finally, we note that in paradigm 1, zero temperature implies zero entropy. This is not true in paradigm 2, since SMCS states have zero temperature but its entropy may take any value.

Some constant temperature surfaces in both paradigms are shown in Fig. (10).

\section{Heat capacity}

As usual, we define the heat capacity as the partial derivative of the energy with respect to temperature, in a zero-work process.

\section{Paradigm 1}

The derivation in Alicki's theoretical framework was performed in Ref. [19],

$$
\mathcal{C}_{\varepsilon}=\frac{k_{B} B\left(1-B^{2}\right)\left[\tanh ^{-1}(B)\right]^{2}(\vec{B} . \hat{v})^{2}}{\tanh ^{-1}(B)\left(B^{2}-(\vec{B} \cdot \hat{v})^{2}\right)\left(1-B^{2}\right)+B(\vec{B} . \hat{v})} .
$$

where the consequences of this result are discussed in detail.

\section{Paradigm 2}

In the new approach, the heat capacity is

$$
C_{\hat{B} \cdot \vec{v}}=\left.\frac{\partial E}{\partial T}\right|_{\hat{B} \cdot \vec{v}} .
$$

The evaluation of the above equation requires to express the energy in terms of $T$ and $\hat{B} \cdot \vec{v}$. From Eq. (49):

$$
B=\tanh \left(\frac{\hat{B} \cdot \vec{v}}{k_{B} T}\right),
$$

which, combined with Eq. (24), leads to

$$
E=-(\hat{B} \cdot \vec{v}) \tanh \left(\frac{\hat{B} \cdot \vec{v}}{k_{B} T}\right) .
$$

Therefore,

$$
C_{\hat{B} . \vec{v}}=k_{B}\left[\frac{x}{\cosh (x)}\right]^{2},
$$

where

$$
x=\frac{\hat{B} \cdot \vec{v}}{k_{B} T},
$$

and $T$ is given by Eq. (49).

\section{Discussion}

A quick inspection of Eqs. (56) and (60) shows that in both cases the heat capacity is non-negative for all possible states. The equivalence between Eq. (56) and the equilibirium heat capacity is shown in Ref. [19]. On the other hand, the expression (60) for the heat capacity in the new paradigm is clearly a more natural extension of the classical result. Since in thermal equilibrium the Bloch vector is parallel to the effective magnetic field,

$$
\hat{B} \cdot \vec{v}=|\vec{v}|=\varepsilon,
$$

and since in that case the temperature of the system equals the environment temperature, Eq. (60) reduces to the well-known expression [42]:

$$
C_{\hat{B} \cdot \vec{v}}=k_{B}\left[\frac{\varepsilon / k_{B} T}{\cosh \left(\varepsilon / k_{B} T\right)}\right]^{2} .
$$

\section{Entropy production}

Classically, the entropy change of a closed system is given by

$$
d S=\frac{\delta Q}{T}+\delta S_{g e n}^{i n t}
$$


where the first term corresponds to the entropy flux through the system's boundary at temperature $T$ due to heat exchange, and the second term is the non-negative entropy production associated to the irreversibilities inside the system [6]. A typical situation is that in which the system is in contact with a heat bath at temperature $T_{E}$. In this case, if the system's temperature and the environment temperature are different, an additional entropy production appears due to the irreversible character of the heat transfer. In this case, the total entropy production can be evaluated by applying Eq. (64) to the system plus its border, so that the irreversible heat transfer occurs in its interior, i.e., considering the environment temperature instead of the system's temperature in Eq. (64). In this case,

$$
d S=\frac{\delta Q}{T_{E}}+\delta S_{g e n}^{t o t}
$$

From the above equations we can make two important observations. One is that the total entropy production can be separated in the internal and the heat transfer contributions. The latter corresponds to the second term in the right-hand side of

$$
\delta S_{g e n}^{t o t}=\delta S_{g e n}^{i n t}+\delta Q\left(\frac{1}{T}-\frac{1}{T_{E}}\right) .
$$

We also note that Eq. (65) can be written as

$$
\delta S_{g e n}^{t o t}=d S-\frac{\delta Q}{T_{E}} .
$$

Note that the two terms on the right-hand side of this equation correspond to the entropy variations of the system and the environment respectively, so the total entropy production and the total entropy variation, in the case of classical systems, coincide:

$$
\delta S_{g e n}^{t o t}=d S^{t o t}
$$

This last equation has represented a big challenge to the possibility of extending Thermodynamics to the quantum regime. Since the evolution of an open system is, in the general case, irreversible, one expects a positive entropy production, $\left(\delta S_{g e n}^{t o t}>0\right)$. However, the unitary evolution of the whole system preserves the density matrix eigenvalues, and, as a consequence the total entropy does not change $\left(d S^{t o t}=0\right)$. This fundamental problem has been addressed in several works [14, 44 46], and it has been suggested that entropy production instead of entropy change is the relevant quantity in order to explain irreversible behavior. In this work, we will only focus in the analysis of Eq. (64) within each paradigm, in order to investigate if an intrinsic entropy production is expected in each case.

\section{Paradigm 1}

This problem has been analyzed in Ref. [19]. From Eqs. (21), (42) and (44), it is straightforward to obtain an equation linking the von Neumann entropy, the heat transferred, and the temperature defined in Alicki's theoretical framework:

$$
d S_{v N}=\frac{\delta \mathcal{Q}}{\mathcal{T}}+\delta \mathcal{S}_{\text {gen }}^{\text {int }}
$$

where the internal entropy production is given by

$$
\delta \mathcal{S}_{\text {gen }}^{\text {int }}=-k_{B} \tanh ^{-1}(B)[\hat{B}-(\hat{v} \cdot \hat{B}) \hat{v}] \cdot d \vec{B}
$$

Since $\hat{B}-(\hat{v} \cdot \hat{B}) \hat{v}$ is orthogonal to $\hat{v}$, for an unitary evolution, or if the system evolves along equilibrium states, Eq. (70) predicts zero internal entropy production, as expected.

\section{Paradigm 2}

From Eqs. (26), 421 and (49),

$$
d S_{v N}=\frac{\delta Q}{T}
$$

and, as a consequence,

$$
\delta S_{\text {gen }}^{\text {int }}=0 .
$$

Thus, in the case of paradigm 2, no intrinsic entropy production is expected in any process. It must be pointed out that this result is valid only in the case of two-level systems. For higher dimensional systems we have been able to find a very reduced set of quantum states for which the concept of local temperature can be consistently defined, but not a generally valid treatment of this quantity [43].

\section{Discussion}

To give a physical interpretation to Eq. (70), we first recall the definition of heat in paradigm 1, Eq. (4),

$$
\delta \mathcal{Q}=-d \vec{B} \cdot \vec{v}
$$

and note that only the part of $d \vec{B}$ which is parallel to $\hat{v}$ is responsible for heat exchange. If we restrict ourselves to the case in which the Hamiltonian is fixed, expressing Eq. (70) in spherical coordinates (with the $z$ axis in the direction of $\hat{v}$ ), we obtain [19]

$$
\delta S_{g e n}^{i n t}=-k_{B} \tanh ^{-1}(B) \sin \theta d(B \sin \theta)
$$

Therefore, the component of $d \vec{B}$ which is orthogonal to $\hat{v}$ and produces no heat, is the one responsible for entropy production. Since $B \sin \theta=C_{l_{1}}$, the entropy produced in paradigm 1 is proportional to the change in the coherence of the qubit in the energy eigenbasis. If coherence is lost, entropy is produced, and destruction of entropy can occur in processes in which the coherence of the qubit 
increases. On the other hand, the non-existence of internally generated entropy in paradigm 2 was expected, since in it heat is defined as the part of the energy change which produces an entropy change. Therefore, in this approach, there are no causes of entropy variation other than the heat flow.

The non-existence of internal entropy production is consistent with the possibility of obtaining an extra amount of work in comparison with the previous approach. Nevertheless, if the system and its environment are at different temperatures, entropy production at the boundary should be expected due to irreversible heat transfer according to Eq. (66),

$$
\delta S_{\text {gen }}^{h t}=\delta Q\left(\frac{1}{T}-\frac{1}{T_{E}}\right) .
$$

However, this result was obtained by subtracting Eqs. (64) and (65), and it was assumed that the heat released by one system equals the one absorbed by the other, an aspect that in the quantum case is not guaranteed in either of the two paradigms. In fact, for a system of two qubits in positive-temperature states, and under a global unitary evolution, the Schmidt decomposition forces both entropy changes to be equal. As a consequence, in paradigm 2 the heat exchanged has the same sign for both systems, so they are releasing or absorbing heat simultaneously.

\section{EXAMPLES}

\section{A. Two-level atom in a heat bath}

In the Markovian approximation (valid in the high temperature limit), the evolution of a two-level atom interacting with a thermal state of the electromagnetic field at temperature $T_{E}$ is given, in the interaction picture, by the master equation [44]

$$
\begin{aligned}
& \frac{\partial \rho_{S}}{\partial t}=\gamma_{0}(\mathcal{N}+1)\left(\sigma_{-} \rho \sigma_{+}-\frac{1}{2} \sigma_{+} \sigma_{-} \rho-\frac{1}{2} \rho \sigma_{+} \sigma_{-}\right) \\
& +\gamma_{0} \mathcal{N}\left(\sigma_{+} \rho \sigma_{-}-\frac{1}{2} \sigma_{-} \sigma_{+} \rho-\frac{1}{2} \rho \sigma_{-} \sigma_{+}\right)
\end{aligned}
$$

where $\gamma_{0}$ is the spontaneous emission rate, $\omega_{0}$ is the transition frequency, $\mathcal{N}$ is the Planck distribution at that frequency,

$$
\mathcal{N}=\frac{1}{e^{\beta_{E} \hbar \omega_{0}}-1},
$$

$\sigma_{ \pm}=\frac{1}{2}\left(\sigma_{x} \pm i \sigma_{y}\right)$, and $\beta_{E}=\left(k_{B} T_{E}\right)^{-1}$.

It is known that in the asymptotic regime, the equilibrium state of the atom is described by the thermal reduced density matrix,

$$
\rho^{e q}=\frac{e^{-\beta_{E} H}}{\operatorname{tr}\left(e^{-\beta_{E} H}\right)},
$$
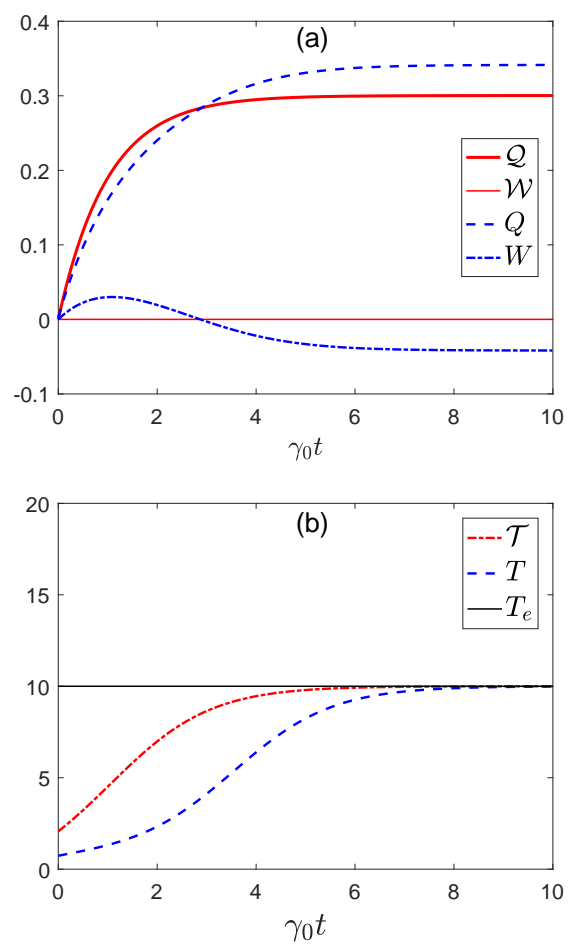

FIG. 2: Comparative evolution of the thermodynamic quantities in both paradigms, for two-level atom interacting with a thermal electromagnetic field at temperature $k_{B} T_{e} / \varepsilon=10$. In panel (a) we show the heat and work exchanged, in panel (b) the temperature. In both cases the initial state is a product state, where the atom's initial density matrix is defined by the Bloch vector $\vec{B}=(0.2,0.5,0.4)$.

which implies that the Bloch vector points in the direction of the effective magnetic field, with modulus

$$
B^{e q}=\tanh \left(\beta_{E} \varepsilon\right),
$$

where $\varepsilon=|\vec{v}|$ is the eigenenergy of the system. As a consequence, the environment temperature determines the equilibrium values of all the thermodynamic quantities. In particular, the equilibrium temperature coincides with the environment temperature.

In the case of paradigm 1 , the total energy variation of the atom corresponds to the heat exchanged with the environment, $\Delta E=\mathcal{Q}$, represented by the thick red continuous line in Fig. 2(a).

From the new perspective of paradigm 2, the thermalization process is related to two different phenomena. On the one hand, since the initial entropy of the atom is arbitrary and its final entropy is defined by the environment temperature, a heat exchange is needed so that the final entropy is the one that ensures thermal equilibrium with the environment. On the other hand, work is also required to rotate the Bloch vector towards the equilibrium direction. Both quantities are also represented in Fig. 2(a). 
The transient positive character of the work observed in the case of paradigm 2 can be understood by analyzing the path towards the equilibrium state in the Bloch sphere, see Fig. 3. Note that when evolution begins, even though both the distance to the $z$ axis, $B \sin \theta$ and $B$ decrease, $\theta$ increases, so work is done on the system. This occurs until the point representing the reduced state reaches the intersection of the trajectory with the tangent line from the center of the sphere (the dashed line in Fig. 3). From that point onwards, both $B$ and $\theta$, and consequently the net work, begin to decrease, resulting, at the end of the thermalization process, in a total negative work done on the system. For this reason, thermalization in the case of paradigm 2 requires greater heat absorption from the environment, part of which is converted into work.

The first part of the process described above shows that although coherence is a useful resource, its consumption does not necessarily imply an extraction of work. In fact, if the temperature of the bath is infinite, there are trajectories that converge to the maximally mixed state tangentially to the plane $z=0$, so a positive total work is performed on the system during the process.

Regarding the behavior of the temperature, in Fig. 2 (b) it is shown that in both theoretical frameworks the temperature increases as the atom absorbs heat. As expected, the respective temperatures tend to the equilibrium temperature, with a faster convergence in Alicki's formulation.

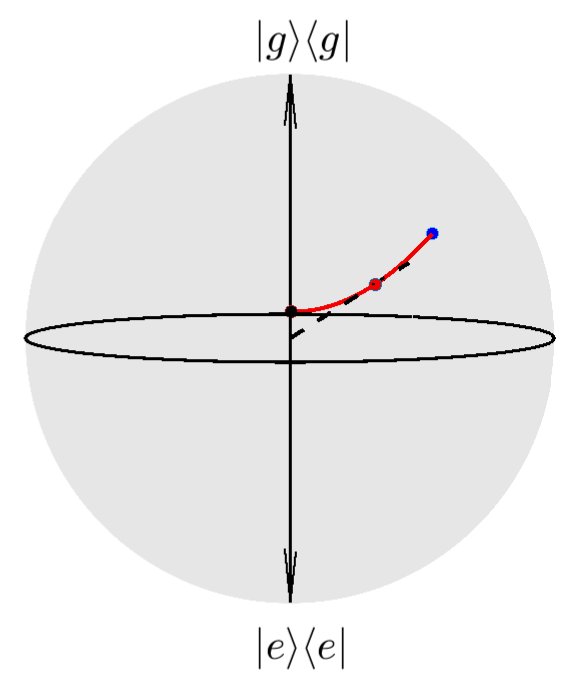

FIG. 3: Evolution of the state of the atom in the Bloch sphere. The system evolves from the initial state $(0.2,0.5,0.4)$ (blue point) towards the thermal state (red point) located at the vertical diameter.

\section{B. Photon exchange between two two-level atoms}

As a second example, let us consider a system composed by two two-level atoms embedded in a common environment at zero temperature. If the atoms are separated a distance $R$ and only the spontaneous emission is taken into account, the system undergoes a dissipative process described by the master equation [47]

$$
\frac{\partial \rho}{\partial t}=\frac{1}{2} \sum_{k, l=A, B} \gamma_{k l}\left(2 \sigma_{-}^{k} \rho \sigma_{+}^{l}-\sigma_{+}^{k} \sigma_{-}^{l} \rho-\rho \sigma_{+}^{k} \sigma_{-}^{l}\right),
$$

where

$$
\sigma_{ \pm}^{A}=\sigma_{ \pm} \otimes \mathbb{I}_{2}, \quad \sigma_{ \pm}^{B}=\mathbb{I}_{2} \otimes \sigma_{ \pm},
$$

$\gamma_{A A}=\gamma_{B B}=\gamma_{0}$ is the spontaneous emission rate of each atom, $\gamma_{A B}=\gamma_{B A}=\gamma=g(R) \gamma_{0} \leq \gamma_{0}$ is the photon-exchange relaxation constant, and $g(R)$ is a function which approaches the value 1 as $R \rightarrow 0$.

In the case $\gamma<\gamma_{0}$, the atoms are not capable of absorbing all the energy emitted by the other, so independently of the initial state, the composed system asymptotically relaxes towards the ground state $|0\rangle \otimes|0\rangle$.

Let us analyze the case in which the system starts from an initially uncorrelated state, with the atom (A) in the partially excited state defined by the Bloch vector $\vec{B}_{A}=$ $(0,0.5,0.8)$, while atom $\mathrm{B}$ is in the ground state, $\vec{B}_{B}=$ $(0,0,1)$.

Since the local Hamiltonians are constant in time, from the point of view of paradigm 1 the emission and absorption of photons is modeled as a heat transfer process between the atoms, with some heat released to the environment. The heat exchanged by each atom is represented by the thick continuous red lines in Figs. 4(a) and 4(b). We note that in the net balance, the atom $\mathrm{A}$ is always releasing heat but at a decreasing rate as it approaches the ground state, while atom B undergoes the process described in the previous paragraph, interpreting its energy change exclusively as heat absorbed and released.

From the perspective of paradigm 2, the energy variation of atom $\mathrm{A}$ includes a negative work component, i.e. work performed by the system, represented by the dashdot blue line in Fig. 4(a). This is due to the fact that the angle formed by the Bloch vector and the vertical direction decreases monotonically in time as the atom approaches the ground state. As a consequence, the amount of heat emitted by the atom is less than in paradigm 1 .

Regarding atom 2, we note that in the first part of the evolution the photon absorption has two effects: it leads to an increase in entropy, interpreted as heat entering the system. In fact, we note in Fig. 4(c) that the temperature also increases. Besides, it also leads to a change in the direction of the Bloch vector, which moves away from the vertical direction. This motion requires external rotational work to overcome its tendency to stay in the vertical direction due to the presence of the magnetic field. We also note that the signs of heat and work are in phase. 
As the system subsequently evolves from state $2 B$ to $3 B$, the emission of photons governing the process comes from the hand of heat released and work done, in amounts opposite to those of the process $1 B \rightarrow 2 B$, with a decrease in temperature. It is also interesting to note that the state $2 B$ occurs approximately when the temperatures of the atoms are equal, so it is reasonable to think that, from that moment on, the energy flows between the atoms balance, and both atoms cool releasing energy to the environment, as it can be seen in Fig. 4
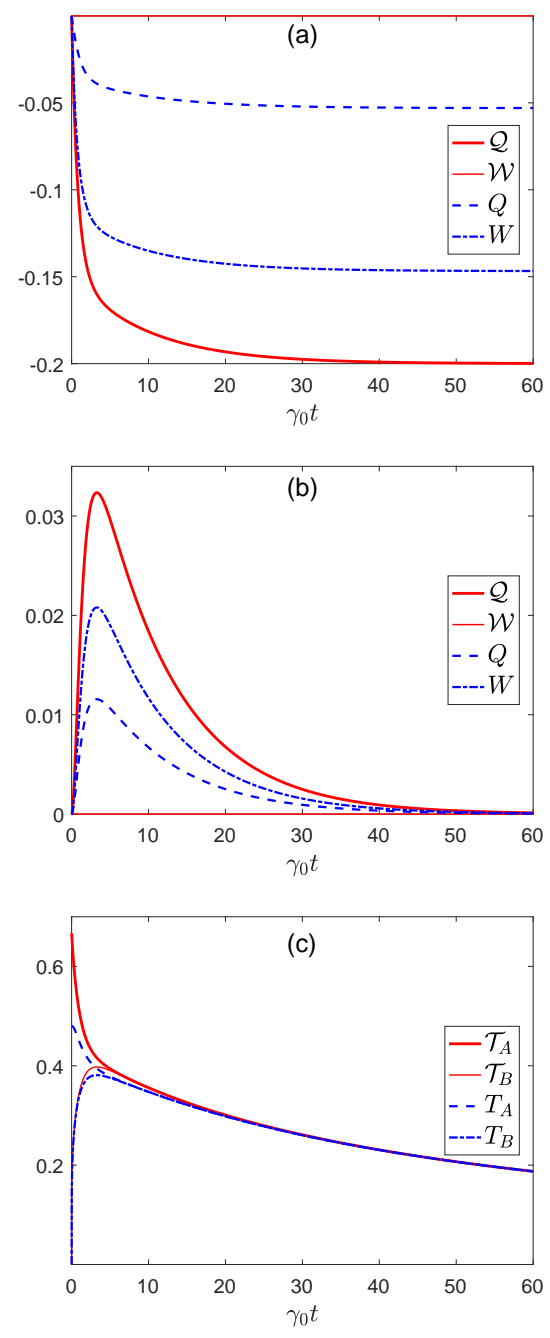

FIG. 4: Thermodynamic quantities for the evolutions of the two atoms considered in Fig. 5 using the two paradigms considered in this work. In panel (a) are shown the heat and work exchanged by atom A. Panel

(b) shows the same quantities for atom B. The respective temperatures, also in both approaches, are shown inpanel (c) .

In Fig. 5 we show the trajectories followed by the states of both atoms in the Bloch sphere. We observe that as the atom A releases energy, its state evolves from its initial state, $1 A$ towards the ground state $2 A$. On the

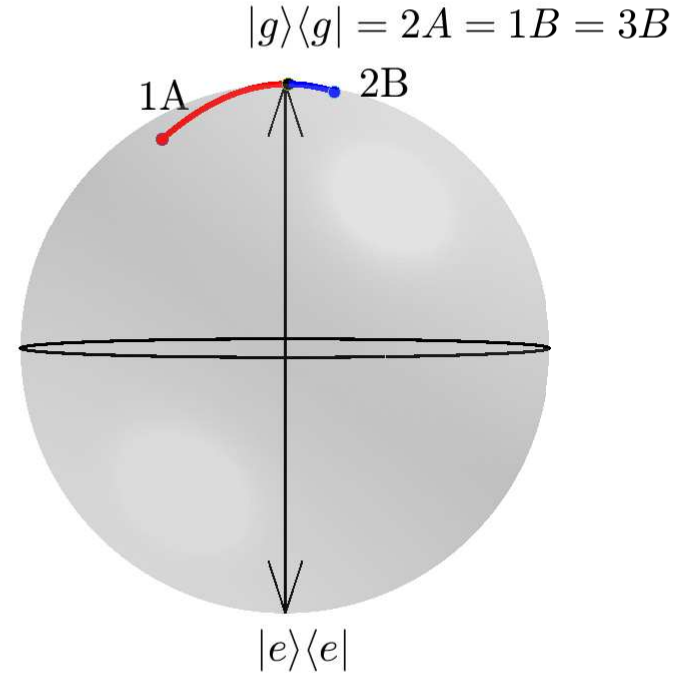

FIG. 5: Trajectories in the Bloch sphere. The initial reduced state is a product state of local densities defined by the Bloch vectors $\vec{B}_{a}=(0,0.5,0.8)$ and $\vec{B}_{b}=(0,0,1)$, and $g(R)=\gamma / \gamma_{0}=0.8$. The environment temperature is zero.

other hand, since atom B starts in the unexcited state $1 B$, it initially absorbs energy, which drives it out of the ground state. Upon reaching the point $2 B$, the energy emitted equals the energy absorbed, and, from that moment on, emission exceeds absorption and the atom relaxes to the ground state $(3 B)$. In the following we will interpret these facts, within the framework of each paradigm.

\section{FINAL REMARKS AND CONCLUSIONS}

In this work we have explored a new proposal about the nature of heat and work in the quantum regime, explicitly developing the comparison, for the simple case of a two-level system, of the new definitions with one of the more accepted paradigms involving these quantities. In addition to making possible to reproduce all the classic results for incoherent states, the predictions based on the new approach present several advantages with respect to the standard paradigm. In particular, the new concept of heat is closer to the classical one, according to which heat is the part of the energy exchange that involves a change in the entropy of the system.

In regard to the definition of work, the main difference in relation to the previous paradigm is the possibility of obtaining work even if the local Hamiltonian of the 
system does not vary in time. This is consistent with situations in which the interaction with the environment is time-dependent, so work is expected to be performed on the system.

In addition, the new paradigm allows to classify the work in two contributions: one associated to Hamiltonian driving, which coincides with the previous definition, and an additional one, associated to the coherence of the state of the system. The latter, that does not appear as work in the first paradigm, is related to the work associated to the rotation of the spin direction. This contribution is analogous to the classical work required to rotate a magnetic dipole in an external magnetic field.

Another important advantage of the new definition of work is that it highlights the importance of coherence as a resource, an aspect already reported in numerous references. In particular, the analysis of a pure dephasing process from the new perspective allows us to understand in a new way why the ability to perform work decreases as the system evolves to the passive state.

A remarkable prediction of the new paradigm is that the thermalization process can no longer be understood as been a purely thermal process, but rather involves a mechanical component associated to the change in orientation of the Bloch vector, or, in the general case, to a rotation of the eigenstates of the system. However, we must not forget that the notion of heat arises due to the impossibility of accessing the microscopic degrees of freedom of macroscopic systems. It is intuitive that, in the absence of that limitation, many energy exchange processes could be exclusively associated to the concept of work. In the new paradigm, heat is linked to a more fundamental inaccessibility, which is a consequence of the quantum description of open systems in terms of mixed states.

Finally, a counterintuitive aspect of paradigm 1 that is not resolved by the new proposal has to do with the fact that, since they are defined using local variables, heat and work cannot be considered energy flows in a strict sense. In the general case, the heat released by one part of the system can be different from the heat absorbed by the other, and the same occurs with the work. Maybe these concepts can be defined unambiguously, and respecting all the intuitive requirements, only in particular situations, such as those in which the system of interest interacts with systems which, by construction, can only exchange either heat or work in the classical sense.

\section{ACKNOWLEDGMENTS}

This work was partially supported by Comisión Académica de Posgrado (CAP), Agencia Nacional de Investigación e Innovación (ANII) and Programa de Desarrollo de las Ciencias Básicas (PEDECIBA) (Uruguay). The authors thank Borhan Ahmadi for stimulating discussions.
[1] S. Carnot, Reflections on the Motive Power of Fire and on Machines Fitted to Develop that Power, Paris: Bachelier (1824).

[2] R. Kosloff, Entropy 15, 2100 (2013).

[3] R.E. Sonntag, C. Borgnakke and G.J. Van Wylen, Fundamentals of Thermodynamics (John Wiley, New York, 2003).

[4] M.W. Zemansky and R.H. Dittman, Heat and Thermodynamics (McGraw-Hill, Auckland, 1997).

[5] H.B. Callen, Thermodynamics and an Introduction to Thermostatistics (John Wiley, New York, 1985).

[6] Y.A. Cengel, and M.A. Boles, Thermodynamics: An Engineering Approach, 4th edition, 1056 pp. ( McGraw-Hill, New York, 2001).

[7] C. M. Bender, D. C. Brody, and B. K. Meister, J. Phys. A: Math. Gen. 33, 4427 (2000).

[8] C. Jarzynski, J. Stat. Mech.: Theo. Exp., 2004, P09005 (2004).

[9] R. Uzdin, A. Levy, and R. Kosloff, Phys. Rev. X 5, 031044 (2015).

[10] M. N. Bera, A. Riera, M. Lewenstein, and A. Winter, Nature Commun. 8, 2180 (2017).

[11] M Horodecki, J Oppenheim, Nat. Commun. 4, 2059 (2013).

[12] P. Skrzypczyk, A. Short and J. Popescu, Nat. Commun. 5, 4185 (2014).

[13] R. Alicki, J. Phys. A: Math. Gen. 12, L103 (1979).
[14] S. Deffner and E. Lutz, Phys. Rev. Lett. 107, 140404 (2011).

[15] R. Alicki, M. Horodecki, P. Horodecki, and R. Horodecki, Open Syst. Inf. Dyn. 11, 205 (2004).

[16] B. Ahmadi, S. Salimi, and A. S. Khorashadl, arXiv:1912.01983 [quant-ph].

[17] S. Alipour, A. Chenu, A. T. Rezakhani, and A. del Campo, arXiv:1912.01939 [quant-ph].

[18] M.A. Nielsen and I.L. Chuang, Quantum Computation and Quantum Information (Cambridge University Press, Cambridge, 2000).

[19] A. Vallejo, A. Romanelli and R. Donangelo, Phys. Rev. E 101, 042132 (2020).

[20] S. Su, J. Chen, Y. Ma, J. Chen, and C. Sun, Chin. Phys. B 27, 060502 (2018).

[21] G. Francica, J. Goold, and F. Plastina, Phys. Rev. E 99, 042105 (2019).

[22] T. Baumgratz, M. Cramer, and M. B. Plenio, Phys. Rev. Lett. 113, 140401 (2014).

[23] M. O. Scully, M. S. Zubairy, G. S. Agarwal, and H. Walther, Science 299, 862 (2003).

[24] K. Korzekwa, M. Lostaglio, J. Oppenheim, and D. Jennings, New J. Phys. 18 (2), 023045 (2016).

[25] P. Abiuso, H. J. D. Miller, M. Perarnau-Llobet, and M. Scandi, Entropy 22, 1076 (2020).

[26] L. Szilard, L. D. Über, Zeitschrift für Physik 53, 840-856 (1929). 
[27] S. Toyabe, T. Sagawa, M. Ueda, E. Muneyuki, and M. Sano, Nature Phys. 6:988 (2010).

[28] J. P. S. Peterson, R. S. Sarthour, A. M. Souza, I. S. Oliveira, J. Goold, K. Modi, D. O. SoaresPinto, and L. C. C'eleri, Proc. Royal Soc. A, 472(2188) 2016.

[29] J. Gemmer, M. Michel, and G. Mahler, Quantum Thermodynamics: The Emergence of Thermodynamic Behavior within Composite Quantum Systems, Lecture Notes in Physics, Springer (2004).

[30] R. S. Johal, Phys. Rev. E, 80, 041119 (2009).

[31] D. Poilblanc, Phys. Rev. Lett. 105, 077202 (2010).

[32] N. S. Williams, K. Le Hur, and A. N. Jordan, J. Phys. A: Math. Theor., 44385003 (2011).

[33] C. L. Latune, I. Sinayskiy and F. Petruccione, Quantum Science and Technology 4 (2), 025005 (2019).

[34] N. Brunner, N. Linden, S. Popescu, and P. Skrzypczyk, Phys. Rev. E 85, 051117 (2012).

[35] A. Manatuly, W. Niedenzu, R. Rom'an-Ancheyta, B. Cakmak, O. E. Mustecaplio glu, and G. Kurizki, Phys. Rev. E 99, 042145 (2019).

[36] R. Román-Ancheyta, B. Çakmak, and Ö. E. Müstecaplioğlu, Quantum Science and Technology 5, 015003 (2019).
[37] M. M. Ali and W. M. Zhang, W. M Zhang, Sci. Rep. 10, 13500 (2020).

[38] M. H. Partovi, Phys. Rev. E 77, 021110 (2008).

[39] K. Micadei, J. P. S. Peterson, A. M. Souza, R. S. Sarthour, I. S. Oliveira, G. T. Landi, T. B. Batalhao, R. M. Serra, and E. Lutz, Nat. Commun. 10, 2456 (2019).

[40] S. R. A. Salinas, Introduction to Statistical Physics (Springer, New York, 2001).

[41] R.K. Pathria, Statistical mechanics (Oxford University press, Oxford, 1996).

[42] S. Mahdavifar and A. Akbari, J. Phys. Soc. Japan 77, 024710 (2008).

[43] A. Vallejo, A. Romanelli, R. Donangelo, incoming.

[44] H.-P. Breuer and F. Petruccione, The Theory of Open Quantum Systems (Oxford University Press, Oxford, 2002).

[45] M. Esposito, K Lindenberg, C. Van den Broeck, New J. Phys., 12, 013013 (2010).

[46] M. Brunelli, L. Fusco, R. Landig, W. Wieczorek, J. Hoelscher-Obermaier, G. Landi, F. L. Semiao, A. Ferraro, N. Kiesel, T. Donner, G. De Chiara, and M. Paternostro, Phys. Rev. Lett., 121, 160604 (2018).

[47] L. Jakóbczyk, A. Jamróz, Phys. Lett. A 318, 318 (2003). 